R. Buccheri et al. (eds.); Endophysics, Time, Quantum and the Subjective; 409-426

(C) 2005 World Scientific Publishing Co. All rights reserved.

\title{
ABSTRACT ALGEBRA, PROJECTIVE GEOMETRY AND TIME ENCODING OF QUANTUM INFORMATION
}

\author{
MICHEL PLANAT \\ FEMTO-ST, University of Franche-Comté, 32 Avenue de l'Observatoire \\ 25044, Besançon, France \\ (planat@lpmo.edu) \\ METOD SANIGA \\ Astronomical Institute, Slovak Academy of Sciences \\ SK-05960 Tatranská Lomnica, Slovak Republic \\ (msaniga@astro.sk)
}

\begin{abstract}
Algebraic geometrical concepts are playing an increasing role in quantum applications such as coding, cryptography, tomography and computing. We point out here the prominent role played by Galois fields viewed as cyclotomic extensions of the integers modulo a prime characteristic $p$. They can be used to generate efficient cyclic encoding, for transmitting secrete quantum keys, for quantum state recovery and for error correction in quantum computing. Finite projective planes and their generalization are the geometric counterpart to cyclotomic concepts, their coordinatization involves Galois fields, and they have been used repetitively for enciphering and coding. Finally, the characters over Galois fields are fundamental for generating complete sets of mutually unbiased bases, a generic concept of quantum information processing and quantum entanglement. Gauss sums over Galois fields ensure minimum uncertainty under such protocols. Some Galois rings which are cyclotomic extensions of the integers modulo 4 are also becoming fashionable for their role in time encoding and mutual unbiasedness.
\end{abstract}

Keywords: Time - Codes - Quantum Information - Galois Fields - Finite Geometry

\section{Introduction}

Many objects of our today life would not have been designed without the revolution of knowledge undertaken one century ago: quantum mechanics. But many philosophers, as well as scientists, are still not satisfied with its abstract interpretation of the physical world. The operational formalism of quantum mechanics can answer almost every question about the observable quantities, but we would like to know more about the quantum machine. We had time and space in the old continuous machinery of the nineteenth 
century physics; where do they reside now? According to the Heisenberg indeterminacy principle, there are gaps in our time description of quantum processes that we cannot fill: accuracy in the isolation of time events means a lack of knowledge of their energy. The same for position in space of a particle which is complementary to the momentum. Some scholars are convinced that we, as humans, are partly responsible for tiny impacts such a particle may suffer during an experiment. The loose of realism would be inherent to the realm of quantum mechanics.

Let us point out that more knowledge about quantum processes may be obtained thanks to quantum information theory - the recent marriage of quantum mechanics and information theory. In the last decade new concepts with quantum bits (qubits), such as qu-cryptography, quteleportation, qu-cloning, qu-computing and qu-money have been implemented [1]. They have grown upon a big stone erected in 1935: the EPR paradox about the entanglement of quantum states. Qubit entanglement (and its generalization to qudits, i.e. many-level quantum states) is the main resource of the newly emerged quantum information technology.

The goal of this paper is to revisit some of the objects of quantum information theory using finite algebraic geometrical concepts such as finite fields (also known as Galois fields), and to give them a geometrical setting. In doing so, a kind of discrete space-time emerges, time being connected to algebraic ideals and space to finite geometries. The notion of a character maps elements of the Galois fields to the quantum states of interest.

\section{Time and Its Relation to Ideals}

\subsection{Ideals and the residue class ring}

Let us start our quest of the nature of time in the algebraic world. Our objects are elements of a finite set which is a ring, $\mathcal{R}$, i.e. the set endowed with two operations "+" and ".". The ring is a group with respect to addition; the product of two elements is in $\mathcal{R}$ and it is both associative and distributive with respect to addition. One needs the concept of an ideal $\mathcal{I}$ in $\mathcal{R}$, denoted $\mathcal{I} \triangleleft \mathcal{R}$, which is a subset of $\mathcal{R}$ such that $\forall a \in \mathcal{I}$, $\forall r \in \mathcal{R}$ one has both $a r \in \mathcal{I}$ and $r a \in \mathcal{I}$. In other words, with the concept of an ideal one pins each element of $\mathcal{R}$ into the subset $\mathcal{I}$; and with the concept of a principal ideal $\mathcal{I}=(a)$, a single element $a$ generates the whole ideal. For a commutative ring $\mathcal{R}$ with an identity the definition is: $(a)=a \mathcal{R}=\{a r, r \in \mathcal{R}\}$. A familiar example is the ring of integers $\mathcal{R}=\mathcal{Z}=\{\cdots,-2,-1,0,+1,+2, \cdots\}$. The principal ideal generated by the number $a=3$ in $\mathcal{Z}$ is $(a)=\{\cdots,-6,-3,0,3,6, \cdots\}$. 
The next important object is the concept of a residue class of $a$ modulo $\mathcal{I}$, which consists of all elements $[a]=\{a+c, \forall c \in \mathcal{I}\}$ and is useful to partition the ring into disjoint classes (or cosets). The set of classes has the property to be a ring, called the residue class ring and denoted $\mathcal{R} / \mathcal{I}$. For the integers $\mathcal{Z}$ modulo the ideal (3), one gets the three classes $[0]=0+(3)$, $[1]=1+(3)$ and $[2]=2+(3)$, and the residue class ring is $\mathcal{Z} /(3)=F_{3}$, where $F_{3}$ is the unique field with 3 elements. It is known that for a prime number $p, Z /(p)=\mathcal{Z}_{p}=F_{p}$, where $\mathcal{Z}_{p}$ is the set of integers modulo $p$ and $F_{p}$ the field with $p$ elements. But, for example, $Z /(4)$ is not a field since $2.2=4=0$ and thus 2 divides 0 .

\subsection{Polynomial rings, Galois fields and their representations}

Let us now consider a ring $\mathcal{R}[x]$ of polynomials with coefficients in $\mathcal{R}$

$$
\mathcal{R}[x]=\left\{a_{0}+a_{1} x+\cdots+a_{n} x^{n}\right\}, \quad a_{i} \in \mathcal{R} .
$$

One says that $g \in \mathcal{R}[x]$ is irreducible if it cannot be factored in $\mathcal{R}$; e.g. $x^{2}-2 \in \mathcal{Q}[x]$ is irreducible in the field $\mathcal{Q}$ of rational numbers, but $x^{2}-2=$ $(x+\sqrt{2})(x-\sqrt{2})$ over the real numbers $\Re$. There is an important theorem that for any polynomial $g \in \mathcal{R}[x]$, the residue class $\operatorname{ring} \mathcal{R}[x] /(g)$ is a field if and only if (iff) $g$ is irreducible over $\mathcal{R}[2]$. For example for $\mathcal{R}=F_{2}=\{0,1\}$, the field with two elements, and since $g=x^{2}+x+1$ is irreducible over $F_{2}$, then $F_{4}=F_{2}[x] /(g)$ is the Galois field with 4 elements $[0]=(g),[1],[x]$ and $[x+1]$. For example $[x]+[x+1]=x+(g)+x+1+(g)=2 x+1+(g)+(g)=$ $1+(g)=[1]$. Similarly $[x][x]=(x+(g))(x+(g))=x^{2}+(g)(2 x+1)=$ $x^{2}+(g)=x^{2}-\left(x^{2}+x+1\right)+(g)=-(x+1)+(g)=(x+1)+(g)=[x+1]$.

It can be shown that a Galois field with $q$ elements exists iff $q=p^{m}$, a power of a prime number $p$. Actually, there are several representations of Galois fields. The first one is as a polynomial as in (11). The second one consists of identifying the Galois field $F_{q}$, with $q=p^{m}$, to the vector space $F_{p}^{m}$ build from the coefficients of the polynomial. The third one uses the property that $F_{q}^{*}=F_{q}-\{0\}$ is a multiplicative cyclic group. One needs the concept of a primitive polynomial. A (monic) primitive polynomial, of degree $m$, in the ring $F_{q}[x]$ is irreducible over $F_{q}$ and has a root $\alpha \in F_{q^{m}}$ that generates the multiplicative group of $F_{q^{m}}$. A polynomial $g \in F_{q}[x]$ of degree $m$ is primitive iff $g(0) \neq 0$ and divides $x^{r}-1$, with $r=q^{m}-1$.

For example, $F_{8}$ can be build from $\mathcal{R}=F_{2}$ and $g=x^{3}+x+1$ which is primitive over $F_{2}$. One gets $F_{8}=F_{2}[x] /(g)=\left\{0,1, \alpha, \alpha^{2}, \alpha^{3}=1+\alpha, \alpha^{4}=\right.$ $\left.\alpha+\alpha^{2}, \alpha^{5}=1+\alpha+\alpha^{2}, \alpha^{6}=1+\alpha^{2}\right\}$, see Table 1 . 
Table 1. Representations of the elements of the Galois field $G F(8)$.

\begin{tabular}{|c|c|c|}
\hline as powers of $\alpha$ & as polynomials & as 3-tuples in $\mathcal{Z}_{2}^{3}$ \\
\hline \hline 0 & 0 & $(0,0,0)$ \\
\hline 1 & 1 & $(0,0,1)$ \\
\hline$\alpha$ & $\alpha$ & $(0,1,0)$ \\
\hline$\alpha^{2}$ & $\alpha^{2}$ & $(1,0,0)$ \\
\hline$\alpha^{3}$ & $1+\alpha$ & $(0,1,1)$ \\
\hline$\alpha^{4}$ & $\alpha+\alpha^{2}$ & $(1,1,0)$ \\
\hline$\alpha^{5}$ & $1+\alpha+\alpha^{2}$ & $(1,1,1)$ \\
\hline$\alpha^{6}$ & $1+\alpha^{2}$ & $(1,0,1)$ \\
\hline
\end{tabular}

\subsection{Cyclic codes as ideals}

In one of our recent papers arithmetical functions were considered relevant models of time evolution [3. For instance, the function $a(n)$ defined as 1 if $n=p^{m}, p$ a prime number, and 0 otherwise, was found to play an important role in the study of phase fluctuations in an oscillator. Three generic functions are met in elementary analytical number theory. One is the Mangoldt function, closely related to the above defined $a(n)$ function. The second is the Euler (totient) function $\phi(n)$, which counts the number of irreducible fractions $l / n, \operatorname{gcd}(l, n)=1$. If one knows the decomposition $n=\prod_{i} p_{i}^{m_{i}}$ as a product of prime powers, then $\phi(n)=n \prod_{i}\left(1-1 / p_{i}\right)$. The third one, the Möbius function, codes the distribution of primes as $\mu(1)=0, \mu(n)=0$ if $n$ contains a square and $(-1)^{k}$ if $n$ is the product of $k$ distinct prime numbers. The last two functions still appear in the theory of cyclic codes, as it will be illustrated below.

In Sect. 2.2 we defined Galois fields as the residue class ring over a ground field $F_{p}$ of characteristic $p$, generated by a polynomial $g(x)$ irreducible over $F_{p}$. One can generalize this view by considering $F_{q}, q=p^{n}$, as the ground field and by defining an ideal $(g)$ from a polynomial $g$ which is irreducible over the polynomial field $F_{q}[x] \equiv F_{q}^{n}$. This definition encompasses all linear cyclic codes. A linear code is any vector subspace of $F_{q}^{n}$, and it is cyclic if one goes from one line to the other of the generating matrix by a shift of its elements.

All cyclic codes are constructed by all the divisors $g$ in $F_{q}[x]$ of the polynomial $x^{n}-1$. The divisors are $g=Q_{d}$, the so-called $d^{\text {th }}$ cyclotomic polynomials, their degree is $\phi(d)$ and they are defined as

$$
Q_{d}=\prod_{d \mid n}\left(x^{d}-1\right)^{\mu(n / d)} .
$$


The Mangoldt function [3] is a way of encoding the primes. ${ }^{a}$ In the new context of cyclic codes, the cyclotomic polynomial also encodes the irregularity of primes. There exists a "zeta" function and a "Riemann hypothesis" for $F_{q}$; the latter was proved by Weil in 1948 [4].

Let us describe a linear $[n, k]$ code from its generator matrix. We use the polynomial

$$
g=g_{0}+g_{1} x+\cdots+g_{m} x^{m} \in F_{q}[x]=F_{q}^{n}, \quad g \mid\left(x^{n}-1\right), \quad \operatorname{deg}(g)=m<n .
$$

The generator matrix is as follows

$$
\left[\begin{array}{ccccccc}
g_{0} & g_{1} & \cdots & g_{m} & 0 & \cdots & 0 \\
0 & g_{0} & \cdots & g_{m-1} & g_{m} & \cdots & 0 \\
\cdots & \cdots & \cdots & \cdots & \cdots & \cdots & \cdots \\
0 & 0 & \cdots & g_{0} & g_{1} & \cdots & g_{m}
\end{array}\right]=\left[\begin{array}{c}
g \\
x g \\
\cdots \\
x^{k-1} g
\end{array}\right]
$$

As an example, we mention the binary Hamming code of length $n=7$, which is obtained from $g=x^{3}+x+1$ of coefficients over $F_{2}$ and contains 4 elements which are the lines of the following generating matrix

$$
\left[\begin{array}{lllllll}
1 & 1 & 0 & 1 & 0 & 0 & 0 \\
0 & 1 & 1 & 0 & 1 & 0 & 0 \\
0 & 0 & 1 & 1 & 0 & 1 & 0 \\
0 & 0 & 0 & 1 & 1 & 0 & 1
\end{array}\right]
$$

The index $n$ plays the role of time and the code is thus a 2 -valued time encoded by the cyclotomic polynomial (2).

\section{Quantum States and Their Relation to Additive Characters}

\subsection{The additive characters}

A character $\kappa(g)$ over an abelian group $G$ is a (continuous) map from $G$ to the field of complex numbers $\mathcal{C}$ that is of modulus 1, i.e. such that $|\kappa(g)|=1, g \in G$. Since there are two operations "+" and "." in the field $F_{q}$, one can define two kinds of characters. Multiplicative characters $\psi_{k}(n)=\exp \left(\frac{2 i \pi n k}{q}\right), k=0, \ldots, q-1$, are well known since they constitute the basis for the ordinary discrete Fourier transform. But additive characters introduced below are the ones which are useful to relate to quantum

\footnotetext{
$\overline{a^{a}}$ The Mangoldt function, $\Lambda(n)$, plays a prominent role in the (still unsolved) Riemann hypothesis. $\Lambda(n)$ equals $\ln (p)$ if $n=p^{m}$ and 0 otherwise. Its average value oscillates around 1 and the error term explicitly relies on the pole at $s=1$ of the Riemann zeta function $\zeta(s)=\sum_{\Re(s)>1} n^{-s}, \Re(s)>1$, on the trivial zeros at $s=-2 l, l>0$, of the extended zeta function $\xi(s)=\pi^{-s / 2} \Gamma(s / 2) \zeta(s), \Gamma(s)$ being the Gamma function, and on the Riemann zeros presumably all located on the critical axis $\Re(s)=1 / 2$.
} 
information. One first defines a map from the extended field $F_{q}, q=p^{m}$, to the ground field $F_{p}$ which is called the trace function

$$
\operatorname{tr}(x)=x+x^{p}+\cdots+x^{p^{m-1}} \in F_{p}, \quad \forall x \in F_{q} .
$$

In addition to its property of mapping an element of $F_{q}$ into $F_{p}$, the trace function has the following properties: $\operatorname{tr}(x+y)=\operatorname{tr}(x)+\operatorname{tr}(y), x, y \in F_{q}$; $\operatorname{tr}(a x)=a \operatorname{tr}(x), x \in F_{q}, a \in F_{p} ; \operatorname{tr}(a)=m a, a \in F_{p} ;$ and $\operatorname{tr}\left(x^{q}\right)=$ $\operatorname{tr}(x), x \in F_{q}$. Using (6), an additive character over $F_{q}$ is defined as

$$
\kappa(x)=\omega_{p}^{\operatorname{tr}(x)}, \omega_{p}=\exp \left(\frac{2 i \pi}{p}\right), x \in F_{q} .
$$

It satisfies the following relation: $\kappa(x+y)=\kappa(x) \kappa(y), x, y \in F_{q}$.

\subsection{Quantum states: qubits and qudits}

Well before the development of quantum information theory physicists developed an efficient formalism for working out quantum states. This formalism was born (with Dirac) in the context of the second quantization of a harmonic oscillator. The language of kets $|u\rangle$ and bras $\langle u|$, for $u$ an element of a Hilbert space $\mathcal{H}$, a vector space over the complex numbers $\mathcal{C}$ equipped with a complex-valued inner product $\mathcal{H} \times \mathcal{H} \rightarrow \mathcal{C}$, is still in use today.

Physically, a qubit is an element of a Hilbert space of dimension $2, \mathcal{H}_{2}$; it can represent a spin $1 / 2$, a two-level atomic system, a two-polarisation state, etc. The most general form of a qubit $|\psi\rangle$ is

$$
|\psi\rangle=a|0\rangle+b|1\rangle, \quad|a|^{2}+|b|^{2}=1 \quad a, b \in \mathcal{C} .
$$

In the computational frame of a qubit base $B_{0}=(|0\rangle,|1\rangle)$, we have $|0\rangle=$ $(1,0)$ and $|1\rangle=(0,1)$. The geometry of the qubit is the Bloch sphere [5], with the qubit $|0\rangle$ at the north pole and the qubit $|1\rangle$ at the south one. In what follows we will be interested in qudits, quantum states in a generic, $q$-dimensional Hilbert space $\mathcal{H}_{q}$ defined as $|\psi\rangle=\sum_{k=0}^{q-1} a_{k}|k\rangle, \sum_{k}\left|a_{k}\right|^{2}=1$, $a_{k} \in \mathcal{C}$, although recently the particular cases of $q=2,4$ and 8 received a lot of attention due to their intimate link to Hopf fibrations (see, e.g. [6]).

Another important concept for quantum measurements has recently emerged, the one of a complete set of mutually unbiased bases (MUBs). Besides the concept of an additive character of the Galois field $F_{q}$, MUBs reveal a connection between $F_{q}$ and the structure of Hilbert space $\mathcal{H}_{q}$. Orthogonal bases of a Hilbert space $\mathcal{H}_{q}$ of finite dimension $q$ are mutually 
unbiased if inner products between all possible pairs of vectors of distinct bases equal $1 / \sqrt{q}$. They are also said to be maximally non-commutative in the sense that a measurement over one basis leaves one completely uncertain as to the outcome of a measurement performed over a basis unbiased to the first. For $q=2$, the eigenvectors of ordinary Pauli spin matrices provide the best-known example.

With a complete set of $q+1$ mutually unbiased measurements one can ascertain the density matrix of an ensemble of unknown quantum $q$-states, so that a natural question emerges as which mathematics may provide the construction. It is known that in dimension $q=p^{m}$ the complete sets of mutually unbiased bases (MUBs) result from Fourier analysis over the Galois field $F_{q}$ ( $p$ odd) [7 or a Galois ring $R_{4^{m}}(p$ even) [8].

Constructions of MUBs in odd characteristic ${ }^{b}$ are related to the character sums with polynomial arguments $f(x)$, called Weil sums,

$$
\sum_{x \in F_{q}} \kappa(f(x))
$$

In particular (see theorem 5.38 in [2]), for a polynomial $f(x) \in F_{q}[x]$ of degree $d \geq 1$, with $\operatorname{gcd}(d, q)=1$, one gets $\left|\sum_{x \in F_{q}} \kappa(f(x))\right| \leq(d-1) q^{1 / 2}$. The complete sets of MUBs are obtained as [8, 9]

$$
\left|\theta_{b}^{a}\right\rangle=\frac{1}{\sqrt{q}} \sum_{n \in F_{q}} \psi_{k}(n) \kappa\left(a n^{2}+b n\right)|n\rangle, \quad a, b \in F_{q},
$$

with $\psi_{k}(n)$ and $\kappa(x)$ defined in Sect.3.1. Eq. (10) defines a set of $q$ bases (with index $a$ ) of $q$ vectors (with index $b$ ). Using Weil sums (9) it is easily shown that for $q$ odd the bases are orthogonal and mutually unbiased to each other and to the computational base $\{|0\rangle,|1\rangle, \cdots,|q-1\rangle\}$ as well.

\subsection{Mutually unbiased bases as quantum phase states}

Dirac was the first to attempt a definition of a phase operator by means of an operator amplitude and phase decomposition. In this description the number operator $N$ and the phase operator $\Theta$ are canonically conjugate such that $[N, \Theta]=i$, where [ ] are the commutator brackets, and this equation leads to a number-phase uncertainty relation $\delta N \delta \phi \geq 1 / 2$. Quantum phase states reaching the bound are coherent, or squeezed states. But there is a big problem in defining such a Hermitian quantum phase operator [10] using the familiar Fock states of the quantized electromagnetic field.

\footnotetext{
${ }^{b}$ Fourier analysis and MUBs in even characteristic are studied in Sect. 6.
} 
One way to circumvent the problem is the use of a discrete Hilbert space $\mathcal{H}_{q}$. It was shown [11] that states $(10)$ obtained from a trivial character $\kappa_{0}=1$ are eigenstates of the Hermitian phase operator

$$
\Theta=\sum_{k \in \mathcal{Z}_{q}} \theta_{k}\left|\theta_{k}\right\rangle\left\langle\theta_{k}\right|,
$$

with eigenvalues $\theta_{k}=\theta_{0}+\frac{2 \pi k}{q}, \theta_{0}$ being an arbitrary initial phase. More generally, the MUB states are eigenstates of a "Galois" quantum phase operator 9

$$
\Theta_{\mathrm{Gal}}=\sum_{b \in F_{q}} \theta_{b}\left|\theta_{b}^{a}\right\rangle\left\langle\theta_{b}^{a}\right|, \quad a, b \in F_{q}
$$

with eigenvalues $\theta_{b}=\frac{2 \pi b}{q}$. The operator can be made more explicit when combined with Eq. (10),

$$
\Theta_{\mathrm{Gal}}=\frac{2 \pi}{q^{2}} \sum_{m, n \in F_{q}} \psi_{k}(n-m) \omega_{p}^{\operatorname{tr}\left[a\left(n^{2}-m^{2}\right)\right]} S(n, m)|n\rangle\langle m|,
$$

with $S(n, m)=\sum_{b \in F_{q}} b \omega_{p}^{\operatorname{tr}[b(n-m)]}$. The diagonal matrix elements feature the sums $S(n, n)=q(q-1) / 2$, while for the non-diagonal ones one gets $S(m, n)=\frac{q}{1-\omega_{p}^{t r(m-n)}}$.

\section{Phase Fluctuations: From Ramanujan to Gauss Sums}

In the previous work [12], the near classical regime of a phase-locked oscillator has been studied and its phase fluctuations have been related to the irregularity of the distribution of prime numbers. A quantum model of phase-locking was derived based on operator (11) with an additional assumption that only elements $\left|\theta_{k}^{\prime}\right\rangle$ with $k$ coprime to $q$ were taken into account. As a result, the quantum phase-locking operator

$$
\Theta_{\text {lock }}=\sum_{k} \theta_{k}^{\prime}\left|\theta_{k}^{\prime}\right\rangle\left\langle\theta_{k}^{\prime}\right|
$$

can be evaluated explicitly as

$$
\Theta_{\text {lock }}=\frac{1}{q} \sum_{n, l} c_{q}(n-l)|n\rangle\langle l|,
$$

where $n, l$ range from 0 to $\phi(q), \phi(q)$ being the Euler totient function. The coefficients in the last equation are the so-called Ramanujan sums,

$$
c_{q}(n)=\sum_{\operatorname{gcd}(p, q)=1} \exp \left(2 i \pi \frac{p}{q} n\right)=\frac{\mu\left(q_{1}\right) \phi(q)}{\phi\left(q_{1}\right)}, \quad q_{1}=q / \operatorname{gcd}(q, n),
$$


where $\mu(q)$ stands for the above-introduced Möbius function.

For the evaluation of phase variability of states we considered a pure phase state of the form 12

$$
|f\rangle=\sum_{n \in \mathcal{Z}_{q}} u_{n}|n\rangle, \quad u_{n}=\frac{1}{\sqrt{q}} \exp (i n \beta),
$$

where $\beta$ is a real parameter, and we computed the phase expectation value $\left\langle\Theta_{\text {lock }}\right\rangle=\sum_{k} \theta_{k}^{\prime}\left|\left\langle\theta_{k}^{\prime} \mid f\right\rangle\right|^{2}$ which reads

$$
\left\langle\Theta_{\text {lock }}\right\rangle=\frac{\pi}{q^{2}} \sum_{n, l} c_{q}(l-n) \exp (i \beta(n-l)) .
$$

For $\beta=1$ it was found that $\left\langle\Theta_{\text {lock }}\right\rangle$ has more pronounced peaks at those values of $q$ which are precisely powers of a prime number, and it can be approximated by the normalized Mangoldt function $\pi \Lambda(q) / \ln q$. For $\beta=0$ the expectation value of $\left\langle\Theta_{\text {lock }}\right\rangle$ is much lower. The parameter $\beta$ can be used to minimize the phase uncertainty well below the classical value [12]. Related phase fluctuations, reflecting properties of the distribution of prime numbers, were obtained in the frame of a quantum statistical mechanics of shift and clock operators. This algebra was also found relevant as a model of time perception [13].

Finally, the phase fluctuations arising from the quantum phase states in MUBs are found to be related to Gaussian sums of the form

$$
G(\psi, \kappa)=\sum_{x \in F_{q}^{*}} \psi(x) \kappa(x) .
$$

Using the notation $\psi_{0}$ for a trivial multiplicative character, $\psi=1$, and $\kappa_{0}$ for a trivial additive character, $\kappa=1$, Gaussian sums (19) satisfy $G\left(\psi_{0}, \kappa_{0}\right)=q-1 ; G\left(\psi_{0}, \kappa\right)=-1 ; G\left(\psi, \kappa_{0}\right)=0$ and $|G(\psi, \kappa)|=q^{1 / 2}$ for nontrivial characters $\kappa$ and $\psi$. We need, however, a more general expression

$$
G(\psi, \kappa)=\sum_{x \in F_{q}} \psi(f(x)) \kappa(g(x)),
$$

where $f, g \in F_{q}[x]$, which is found to be of the order of magnitude $\sqrt{q}$ ([2], p. 249). As a matter of fact, the two factors in the expression for the probability distribution $\left\langle\Theta_{\text {Gal }}\right\rangle=\sum_{b \in F_{q}} \theta_{b}\left|\left\langle\theta_{b} \mid f\right\rangle\right|^{2}$ have absolute values bounded by the absolute value of generalized Gauss sums (20), so that $\left|\left\langle\theta_{b} \mid f\right\rangle\right|^{2} \leq \frac{1}{q}$ as it can be expected for an arbitrary phase factor. To be 
more rigorous, the phase expectation value can be expressed as

$$
\left\langle\Theta_{\text {Gal }}\right\rangle=\frac{2 \pi}{q^{3}} \sum_{m, n \in F_{q}} \psi_{k}(m-n) \exp [i(n-m) \beta] \omega_{p}^{\operatorname{tr}\left[a\left(m^{2}-n^{2}\right)\right]} S(m, n),
$$

where $S(m, n)$ was defined in Sect. 3.3. All the $q$ diagonal terms $m=n$ in $\left\langle\Theta_{\text {Gal }}\right\rangle$ contribute an order of magnitude $\frac{2 \pi}{q^{3}} q S(n, n) \simeq \pi$. The contribution of off-diagonal terms and possible cancellation of phase oscillations in the phase expectation value and in phase variance are discussed in [9].

\section{Mutual Unbiasedness and Maximal Entanglement}

As we have shown, there is a founding link between irreducible polynomials over a ground field $F_{p}$ and complete sets of mutually unbiased bases arising from the Fourier transform over a lifted field $F_{q}, q=p^{m}$. On the other hand, the physical concept of entanglement over a Hilbert space $\mathcal{H}_{q}$ evokes irreducibility. Roughly speaking, entangled states in $\mathcal{H}_{q}$ cannot be factored into tensorial products of states in Hilbert spaces of lower dimension. We will now show that there is an intrinsic relation between MUBs and maximal entanglement.

We are all familiar with the Bell states

$$
\begin{aligned}
\left(\left|\mathcal{B}_{0,0}\right\rangle,\left|\mathcal{B}_{0,1}\right\rangle\right) & =\frac{1}{\sqrt{2}}(|00\rangle+|11\rangle,|00\rangle-|11\rangle), \\
\left(\left|\mathcal{B}_{1,0}\right\rangle,\left|\mathcal{B}_{1,1}\right\rangle\right) & =\frac{1}{\sqrt{2}}(|01\rangle+|10\rangle,|01\rangle-|10\rangle),
\end{aligned}
$$

where a compact notation $|00\rangle=|0\rangle \odot|0\rangle,|01\rangle=|0\rangle \odot|1\rangle$, etc. is employed for the tensorial products. These states are both orthonormal and maximally entangled, such that trace $_{2}\left|\mathcal{B}_{h, k}\right\rangle\left\langle\mathcal{B}_{h, k}\right|=\frac{1}{2} I_{2}$, where trace $_{2}$ is the partial trace over the second qubit $[5]$. One can define more generalized Bell states using the multiplicative Fourier transform [11] applied to the tensorial products of two qudits [9], viz.

$$
\left|\mathcal{B}_{h, k}\right\rangle=\frac{1}{\sqrt{q}} \sum_{n=0}^{q-1} \omega_{q}^{k n}|n, n+h\rangle .
$$

These states are both orthonormal, $\left\langle\mathcal{B}_{h, k} \mid \mathcal{B}_{h^{\prime}, k^{\prime}}\right\rangle=\delta_{h h^{\prime}} \delta_{k k^{\prime}}$, and maximally entangled, trace $_{2}\left|\mathcal{B}_{h, k}\right\rangle\left\langle\mathcal{B}_{h, k}\right|=\frac{1}{q} I_{q}$.

For odd characteristic, we can also define a more general class of maximally entangled states, using the Fourier transform over $F_{q}$ and Eq. (10), as follows

$$
\left|\mathcal{B}_{h, b}^{a}\right\rangle=\frac{1}{\sqrt{q}} \sum_{n=0}^{q-1} \omega_{p}^{t r[(a n+b) n]}|n, n+h\rangle .
$$


A list of the generalized Bell states of qutrits for the base $a=0$ can be found in [14], the work that relies on a coherent state formulation of entanglement. In general, for $q$ a power of a prime, starting from (23) one obtains $q^{2}$ bases of $q$ maximally entangled states. Each set of the $q$ bases (with $h$ fixed) has the property of mutual unbiasedness.

\section{Mutually Unbiased Bases in Even Characteristic}

\subsection{Construction of the Galois rings of characteristic four}

The Weil sums (9), which have been proved useful in construction of MUBs in odd characteristic, are not useful for $p=2$ since in this case the degree $d$ of the polynomial $f(x)$ is such that $\operatorname{gcd}(d, q)=2$ - the characteristic of the relevant Galois fields. An elegant method for constructing complete sets of MUBs of $m$-qubits was found in [8]. It makes use of algebraic objects in the context of quaternary codes [22], the so-called Galois rings $R_{4^{m}}$. In contrast to the Galois fields where the ground alphabet has $p$ elements in the field $F_{p}=\mathcal{Z}_{p}$, the ring $R_{4^{m}}$ takes its ground alphabet in $\mathcal{Z}_{4}$. To construct this ring one uses the ideal class $(h)$, where $h$ is a (monic) basic irreducible polynomial of degree $m$ such that its restriction to $\bar{h}(x)=h(x) \bmod 2$ is irreducible over $\mathcal{Z}_{2}$. The Galois ring $R_{4^{m}}$ is the residue class ring $\mathcal{Z}_{4}[x] /(h)$ and has cardinality $4^{m}$.

We also need the concept of a primitive polynomial. To this end, we recall that a (monic) primitive polynomial, of degree $m$, in the ring $F_{q}[x]$ is irreducible over $F_{q}$ and has a root $\alpha \in F_{q^{m}}$ that generates the multiplicative group of $F_{q^{m}}$. A polynomial $f \in F_{q}[x]$ of degree $m$ is primitive iff $f(0) \neq 0$ and divides $x^{r}-1$, where $r=q^{m}-1$. Similarly for Galois rings $R_{4^{m}}$, if $\bar{h}[x]$ is a primitive polynomial of degree $m$ in $\mathcal{Z}_{2}[x]$, then there is a unique basic primitive polynomial $h(x)$ of degree $m$ in $\mathcal{Z}_{4}[x]$ (it divides $x^{r}-1$, with $\left.r=2^{m}-1\right)$. It can be found as follows [9]. Let $\bar{h}(x)=e(x)-d(x)$, where $e(x)$ contains only even powers and $d(x)$ only odd powers; then $h\left(x^{2}\right)= \pm\left(e^{2}(x)-d^{2}(x)\right)$. For $m=2,3$ and 4 one takes $\bar{h}(x)=x^{2}+x+1$, $\bar{h}(x)=x^{3}+x+1$ and $\bar{h}(x)=x^{4}+x+1$ and gets $h(x)=x^{2}+x+1$, $x^{3}+2 x^{2}+x-1$ and $x^{4}+2 x^{2}-x+1$, respectively.

Any non zero element of $F_{p^{m}}$ can be expressed in terms of a single primitive element. This is no longer true in $R_{4^{m}}$, which contains zero divisors. But in the latter case there exists a nonzero element $\xi$ of order $2^{m}-1$ which is a root of the basic primitive polynomial $h(x)$. Any element $y \in R_{4^{m}}$ can be uniquely determined in the form $y=a+2 b$, where $a$ and $b$ belong to the so-called Teichmüller set $\mathcal{T}_{m}=\left(0,1, \xi, \cdots, \xi^{2^{m}-2}\right)$. Moreover, one finds that $a=y^{2^{m}}$. We can also define the generalized trace to the 
base ring $\mathcal{Z}_{4}$ as the map

$$
\widetilde{\operatorname{tr}}(y)=\sum_{k=0}^{m-1} \sigma^{k}(y)
$$

where $\sigma(y)$ is the so-called Frobenius automorphism, obeying the rule

$$
\sigma(a+2 b)=a^{2}+2 b^{2} .
$$

In $R_{4^{m}}$ the additive characters acquire the form

$$
\tilde{\kappa}(x)=\omega_{4}^{\widetilde{\operatorname{tr}}(x)}=i^{\widetilde{\operatorname{tr}}(x)} .
$$

\subsection{Exponential sums over $R_{4^{m}}$}

The Weil sums (9) are replaced by the following exponential sums [8]

$$
\Gamma(y)=\sum_{u \in \mathcal{T}_{m}} \tilde{\kappa}(y u), \quad y \in R_{4^{m}},
$$

which satisfy

$$
|\Gamma(y)|= \begin{cases}0 & \text { if } y \in 2 \mathcal{T}_{m}, y \neq 0, \\ 2^{m} & \text { if } y=0, \\ \sqrt{2^{m}} & \text { otherwise. }\end{cases}
$$

Gauss sums for Galois rings were constructed in [23] and are of the form

$$
G_{y}(\tilde{\psi}, \tilde{\kappa})=\sum_{x \in R_{4} m} \tilde{\psi}(x) \tilde{\kappa}(y x), \quad y \in R_{4^{m}},
$$

where the multiplicative character $\bar{\psi}(x)$ can be made explicit. Using the notation $\tilde{\psi}_{0}$ for a trivial multiplicative character and $\tilde{\kappa_{0}}$ for a trivial additive character, we get $G\left(\tilde{\psi_{0}}, \tilde{\kappa_{0}}\right)=4^{m}, G\left(\tilde{\psi}, \tilde{\kappa_{0}}\right)=0$ and $|G(\tilde{\psi}, \tilde{\kappa})| \leq 2^{m}$.

\subsection{Mutually unbiased bases of m-qubits}

It was mentioned in the previous section that each element $y$ of the ring $R_{4^{m}}$ can be decomposed as $y=a+2 b$, with $a$ and $b$ belonging to the Teichmüller set $\mathcal{T}_{m}$. Employing this fact in the character function $\tilde{\kappa}$, one obtains

$$
\left|\theta_{b}^{a}\right\rangle=\frac{1}{\sqrt{2^{m}}} \sum_{n \in \mathcal{T}_{m}} \tilde{\psi}_{k}(n) \tilde{\kappa}[(a+2 b) n]|n\rangle, \quad a, b \in \mathcal{T}_{m} .
$$

This defines a set of $2^{m}$ bases (with index $a$ ) of $2^{m}$ vectors (with index b). Using Eq. (27), it is easy to show that the bases are orthogonal and 
mutually unbiased to each other and to the computational base. (For the explicit derivation of the bases, see [9].)

Quantum phase states of $m$-qubits (30) derive from a "Galois ring" quantum phase operator as in Eq. (12), and calculations similar to those in Sect. 3.3 can be performed, since the trace operator defined by Eq. (24) obeys the rules similar to those of the field trace operator, Eq. (6). In analogy to the case of qudits in dimension $p^{m}, p$ an odd prime, the phase properties for sets of $m$-qubits rely substantially on Eq. (29). As before, the calculations are tedious, but they can successfully be accomplished in specific cases.

\section{Quantum Geometry From Projective Planes}

We have related complete sets of MUBs in dimension $p^{m}, p$ odd, to additive characters over a Galois field. Complete sets of MUBs offer an intriguing geometrical interpretation, being related to discrete phase spaces 15], finite projective planes [16, 17], convex polytopes [18], and complex projective 2designs [19]. The last-mentioned paper also points out an interesting link to symmetric informationally complete positive operator measures (SICPOVMs) [20] and to Latin squares [21]. We focus here on the relation of MUBs to finite geometries and projective planes.

\subsection{Mutually unbiased bases and projective planes}

A remarkable link between mutually unbiased measurements and finite projective geometry has recently been noticed [16]. Let us find the minimum number of different measurements we need to determine uniquely the state of an ensemble of identical $q$-state particles. The density matrix of such an ensemble, being Hermitean and of unit trace, is specified by $\left(2 q^{2} / 2\right)-1=q^{2}-1$ real parameters. When one performs a non-degenerate orthogonal measurement on each of many copies of such a system one eventually obtains $q-1$ real numbers (the probabilities of all but one of the $q$ possible outcomes). The minimum number of different measurements needed to determine the state uniquely is thus $\left(q^{2}-1\right) /(q-1)=q+1$ [7, 15 .

It is striking that the identical expression can be found within the context of finite projective geometry. A finite projective plane is an incidence structure consisting of points and lines such that any two points lie on just one line, any two lines pass through just one point, and there exist four points, no three of them on a line [24. From these properties it readily follows that for any finite projective plane there exists an integer $q$ with the 


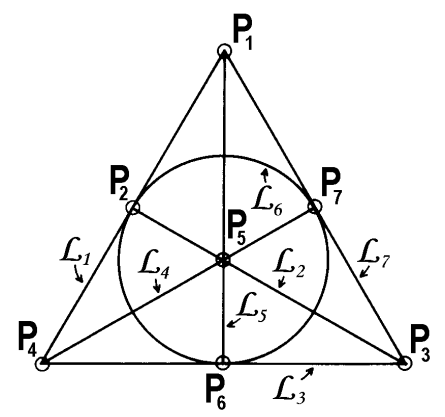

Figure 1. The Fano plane; small circles (denoted as $P_{1}, \ldots, P_{7}$ ) represent its points, while line-segments $\left(\mathcal{L}_{1}, \ldots, \mathcal{L}_{5}\right.$ and $\left.\mathcal{L}_{7}\right)$ and a circle $\left(\mathcal{L}_{6}\right)$ stand for its lines.

properties that any line contains exactly $q+1$ points, any point is the meet of exactly $q+1$ lines, and the number of points is the same as the number of lines, namely $q^{2}+q+1$. This integer $q$ is called the order of the projective plane. The most striking issue here is that the order of known finite projective planes is a power of prime. The question of which other integers occur as orders of finite projective planes remains one of the most challenging problems of contemporary mathematics. The only "no-go" theorem known so far in this respect is the Bruck-Ryser theorem [24] saying that there is no projective plane of order $q$ if $q-1$ or $q-2$ is divisible by 4 and $q$ is not the sum of two squares. Out of the first few non-prime-power numbers, this theorem rules out finite projective planes of order $6,14,21,22,30$ and 33 . Moreover, using massive computer calculations, it was proved that there is no projective plane of order ten. It is surmised that the order of any projective plane is a power of a prime.

It is conjectured 16 that the question of the existence of a set of $q+1$ mutually unbiased bases in a $q$-dimensional Hilbert space if $q$ differs from a power of a prime number is identical with the problem of whether there exist projective planes whose order $q$ is not a power of a prime number.

The smallest projective plane, also called the Fano plane (see Fig. 1), is obviously the $q=2$ one; it contains 7 points and 7 lines, any line contains 3 points and each point is on 3 lines. It may be viewed as a 3 -dimensional vector space over the field $G F(2)$, each point being a triple $\left(g_{1}, g_{2}, g_{3}\right)$, excluding the $(0,0,0)$ one, where $g_{i} \in G F(2)=\{0,1\}$ [24]. The points of this plane can also be represented in terms of the non-zero elements of the Galois field $G=G F\left(2^{3}\right)$, see the last column of Table 1 . 


\subsection{Cyclic codes and projective spaces}

As shown in Sect.2.3, a linear code $C$ is a subspace of $F_{q}^{n}, q=p^{m}$. And a cyclic code is an ideal $(g)$ in the polynomial field $F_{q}[x] \equiv F_{q}^{n}$ attached to a polynomial $g$ irreducible over $F_{q}$. One defines the Hamming distance [2] between $x$ and $y$ in $F_{q}^{n}$ as the number of coordinates in which $x$ and $y$ differ. The minimum distance of a code is an important concept which characterizes its efficiency for error correcting; it is defined as

$$
d=d_{\min }(C)=\min _{\substack{u, v \in C \\ u \neq v}} d(u, v)
$$

A linear code corrects up to $\left[\frac{d-1}{2}\right]$ and detect up to $d-1$ errors. It can be shown that for a linear $[n, k]$ code, the following bound holds

$$
d \leq n-k+1=d_{\max }
$$

A minimum distance code (or a maximum distance separable, MDS code) is such that $d=d_{\max }$ and it is usually referred to as a $[n, k, d]$ code (or $[n, n-r, r+1]$ code). The binary Hamming [7, 4] code introduced in Sect.2.3 thus corrects up to 1 and detect up to 3 errors. It is the MDS $[7,4,4]$ code.

There exists an intimate link between this code and the Fano plane, which can be inferred as follows. Let us take its seven codewords 1 to 7 by cyclic extending of matrix (5), viz.

$$
\left[\begin{array}{lllllll}
1 & 1 & 0 & 1 & 0 & 0 & 0 \\
0 & 1 & 1 & 0 & 1 & 0 & 0 \\
0 & 0 & 1 & 1 & 0 & 1 & 0 \\
0 & 0 & 0 & 1 & 1 & 0 & 1 \\
1 & 0 & 0 & 0 & 1 & 1 & 0 \\
0 & 1 & 0 & 0 & 0 & 1 & 1 \\
1 & 0 & 1 & 0 & 0 & 0 & 1
\end{array}\right] .
$$

The above matrix is nothing but the incidence matrix of the Fano plane, obtained as follows: if $P_{j}$ is the $j$ th point and $\mathcal{L}_{i}$ represents the $i$ th line of the Fano plane, the elements of the matrix are

$$
a_{i j}= \begin{cases}1 & \text { if } P_{j} \in \mathcal{L}_{i} \\ 0 & \text { otherwise }\end{cases}
$$

The link between good codes and projective geometry has recently received considerable attention [24]. Let us define a vector space $V$ of dimension $\delta+1 \geq 3$ over $F_{q}$. Then a projective geometry $P(V)$ can be 
defined as follows. The points of $P(V)$ are its 1-dimensional subspaces, the lines its 2-dimensional subspaces and the incidence structure in $P(V)$ is the set-theoretical containment. The geometry $P(V)$ is the projective space coordinatized by the Galois field $F_{q}$. This $\delta$-dimensional projective space $P(V)$ over $F_{q}$ is usually denoted as $P G(\delta, q)$.

Next, a set of points in $P=P G(\delta, q)$ is called an arc if any $\delta+1$ of its points form a basis of $P$. An arc having $n$ points is called an $n$-arc. In a projective plane $P G(2, q)$ an $n$-arc is a set of $n$ points no three of which are collinear. If each point of an $n$-arc is exactly on one tangent, the arc is called an oval. The maximum value of $n$ for an $n$-arc is

$$
m(2, q)= \begin{cases}q+1 \text { when } q \text { is odd } \\ q+2 \text { when } q \text { is even. }\end{cases}
$$

The meaning of Eq. (35) is as follows. If $q$ is odd, then arcs with a maximum number of points are ovals. If $q$ is even then, each oval can be uniquely extended to a $(q+2)$-arc, which is called a hyperoval. The possible correspondence between ovals and complete set of MUBs is discussed in [17.

There is a one to one correspondence between the generator matrix of $[n, n-r]$ MDS codes and $n$-arcs in $P G(r-1, q)$ (25], p. 73). The construction of good codes with a prescribed minimum distance can be rephrased as follows. One is given the minimum distance $d$ and $r$. Determine the greatest length of the code, $\max _{d-1}(r, q)$.

The simplest case is $d=3$ for which $\max _{2}(r, q)$ is the maximum possible number of points in $P G(r-1, q)$ such that two of them are independent: this is, obviously, the total number of points in $P G(r-1, q)$, and thus

$$
\max _{2}(r, q)=q^{r-1}+\cdots+q+1 \text {. }
$$

In particular, we have $\max _{2}(r, 2)=2^{r}-1$, which corresponds to the Hamming $[n, n-r]$ code. The case $d=4$ is less trivial. Only partial results are known:

$$
\begin{gathered}
\max _{3}(r, 2)=2^{r-1}, \\
\max _{3}(3, q)=\left\{\begin{aligned}
q+1 & \text { when } q \text { is odd, } \\
q+2 & \text { when } q \text { is even, }
\end{aligned}\right.
\end{gathered}
$$

and

$$
\max _{3}(4, q)=q^{2}+1 .
$$

Putting $r=3$ in Eq. (37) one gets the case of the $[7,4,4]$ code considered above. The geometry of Eq. (38) answers to ovals ( $q$ odd) and hyperovals ( $q$ even) of $P G(2, q)$, that of Eq. (39) to ovoids ${ }^{c}$ of $P G(3, q)$.

${ }^{c}$ An ovoid is a nonempty set $\mathcal{O}$ of points of $P G(d, q)$ such that no three points of $\mathcal{O}$ are 


\section{Conclusion}

Let us briefly summarize basic ideas developed in the paper. At the algebraic level, there exists a polynomial field $F_{q}^{n}$ defined over a "base" field $F_{q}$, with $q=p^{m}$ and $p$ a prime. The cyclic encoding (in "time" $n$ ) comes from (cyclotomic) laws of partitioning $F_{q}^{n}$. At the geometrical level, a projective space can always be partioned into subsets (called spreads). For example, $P G(3, q)$ is partioned into subsets of $q^{2}+1$ mutually skew (i.e. pairwise disjoint) lines. These may well form ovoids, which "give rise" to $M D S$-codes. Similarly, there is a partitioning of a projective plane $P G(2, q)$ into sets of $q+1$ lines. These may well be (the tangents of) ovals, which are conjectured to reproduce properties of the sets of mutually unbiased bases. Here, we play with a two-dimensional "quantum space-time." The vectorial projective space, $P G(\delta, q)$, is thus a promising playground for tackling both the measurement and coding problems in quantum mechanics. Yet, there exist more general kinds of finite (projective) geometries, e.g. (non-)Desarguesian projective planes defined over quasi-/near-fields, the latter obeying less stringent rules than fields [26]. These are, we believe, candidates for addressing another intriguing quantum effects like partial entanglement and decoherence.

\section{Acknowledgement}

M.S. would like to acknowledge the support received from a 2004 SSHN Physics Fellowship (\#411867G/P392152B).

\section{References}

1. Bouwmeester, D., Ekert, A.K., and Zeilinger, A. (eds.) (2000) The Physics of Quantum Information, Springer Verlag, Berlin.

2. Lidl, R., and Niederreiter, H. (1983) Finite Fields, Addison-Wesley, Reading (Mass.).

3. Planat, M. (2001) $1 / f$ noise, the measurement of time and number theory, Fluct. Noise Lett. 1, R65-R79.

4. Roquette, P. (2003) The Riemann hypothesis in characteristic $p$, its origin and development, preprint available at http://www.rzuser.uni-heidelberg.de/ ci3/.

5. Nielsen, M.A., and Chuang, I. (2000) Quantum Computation and Quantum Information, Cambridge University Press, Cambridge.

6. Bernevig, B.A., and Chen, H.D. (2003) Geometry of the 3-qubit state, entanglement and division algebras, J. Phys. A: Math. Gen. 36, 8325-8339.

7. Wootters, W.K., and Fields, B.D. (1989) Optimal state-determination by mutually unbiased measurements, Ann. of Phys. 191, 363-381.

collinear and for each point $Q \in \mathcal{O}$ the tangents through $Q$ span the whole hyperplane. 
8. Klappenecker, A., and Rötteler, M. (2004) Constructions of mutually unbiased bases, Lecture Notes in Computer Science 2948, 137-144; preprint quant-ph/0309120.

9. Planat, M., and Rosu, H. (2005) Quantum phase uncertainty in mutually unbiased measurements and Gauss sums, SPIE Proceedings, Fluctuations and Noise 5842, pp. 183-193; preprint quant-ph/0502167.

10. Lynch, R. (1995) The quantum phase, a critical review, Physics Reports 256, 367-436.

11. Pegg, D.T., and Barnett, S.M. (1989) Phase properties of the quantized singlemode electromagnetic field, Phys. Rev. A 15, 1665-1675.

12. Planat, M., and Rosu, H. (2003) Cyclotomy and Ramanujan sums in quantum phase-locking, Phys. Lett. A 315, 1-5.

13. Planat, M. (2004) On the cyclotomic quantum algebra of time perception, NeuroQuantology 2(4), 292-308.

14. Fujii, K. (2001) A relation between coherent states and generalized Bell states, preprint quant-ph/0105077.

15. Gibbons, K.S., Hoffman, M.J., and Wootters, W.K. (2004) Discrete phase space based on finite fields, Phys. Rev. A 70, 062101-062123; preprint quant-ph/0401155.

16. Saniga, M., Planat, M., and Rosu, H. (2004) Mutually unbiased bases and finite projective planes, J. Opt. B: Quantum Semiclass. Opt. 6, L19-L20; preprint math-ph/0403057.

17. Saniga, M., and Planat, M. (2005) Viewing sets of mutually unbiased bases as arcs in finite projective planes, Chaos, Solitons $\& 5$ Fractals 26(5), 1267-1270; preprint quant-ph/0409184.

18. Bengtsson, I., and Ericsson, A. (2004) Mutually unbiased bases and the complementarity polytope, preprint quant-ph/0410120

19. Klappenecker, A., and Rötteler, M. (2005) Mutually unbiased bases are complex projective 2-designs, preprint quant-ph/0502031.

20. Grassl, M. (2004) On SIC-POVMs and MUBs in dimension six, preprint quant-ph/0406175.

21. Wocjan, P., and Beth, T. (2004) New construction of mutually unbiased bases in square dimensions, preprint quant-ph/0407081.

22. Wan, Z.-X. (1997) Quaternary Codes, World Scientific, Singapore.

23. Yunchang, Oh, and Heung-Joon, Oh (2001) Gauss sums over Galois rings of characteristic 4, Kangweon-Kyungki Math. Jour. 9, 1-7.

24. Beutelspacher, A., and Rosenbaum, U. (1998) Projective Geometry: From Foundations to Applications, Cambridge University Press, Cambridge.

25. Hirschfeld, J.W.P. (1998) Projective Geometries Over Finite Fields, Oxford Science Publications, Oxford.

26. Hughes, D.R., and Piper, F.C. (1973) Projective Planes, Springer, New York. 